\title{
Seprafilm Mediated Immune Response Mimic Danger Signal: A Pilot Study in Ovarian Cancer Cases
}

\author{
Tzu-Hsuan Chin', Hsiu-Huei Peng1, Cheng-Tao Lin ${ }^{1,2,3^{*}}$
}

${ }^{1}$ Department of Obstetrics and Gynecology, Chang Gung Memorial Hospital, Chang Gung University College of Medicine, Linkou Medical Center, Taoyuan, Taiwan ${ }^{2}$ Chang Gung Immunology Consortium, Chang Gung Memorial Hospital, Chang Gung University College of Medicine, Linkou Medical Center, Taoyuan, Taiwan

${ }^{3}$ Chang Gung Gynecological Oncology Cancer Center, Chang Gung Memorial Hospital, Linkou Medical Center, Taoyuan, Taiwan

\begin{abstract}
Danger signal was first proposed in 1994. In response to danger signal, dendritic cells, as the director of the T-cell immune system, would be matured and further enhance T-cell response. Hyaluronan had been reported as one of endogenous danger signals. Because of Seprafilm (most used for preventing post-operation adhesion) is also an hyaluronate-based agent, so we choose Seprafilm as our "candidate" of emerging danger signal to increasing T-cell response. We perform a pilot study in 10 ovarian cancer patients to compare the immune risk profiles between cases using Seprafilm (5 cases) and not using Seprafilm (5 cases). We found that there is no statistical difference on the immune risk profiles between two groups. However, a trend of increased CD4 in Seprafilm group was noted, indicating that Seprafilm might be an optional agent mimic danger signal. The potential value of Seprafilm to augment host immunosurveillance to improve survival rate in cancer patients is unknown, further study is needed to clarify the possibility of clinical applications.
\end{abstract}

Keywords: Seprafilm; Immune; Ovarian cancer

\section{Introduction}

Most of the cases of ovarian cancer are diagnosed as advanced stage. Although there is $70 \%$ to $80 \%$ of complete remission after standard treatment (including debulking surgery, chemotherapy and radiotherapy), they still face $60 \%$ to $70 \%$ chance of disease relapse. Once relapse, they suffer from detrimental outcome following first-line treatment [1]. So we urgently need better therapy, and how to augment the host immunosurveillance may be the way forward.

As we know, in response to danger signal, dendritic cells would be matured and further enhance T-cell response. Hyaluronan had been reported as one of endogenous danger signals, so we choose Seprafilm as our "candidate" of emerging danger signal to increasing T-cell response in ovarian cancer patients.

\section{Materials and Methods}

From 2009 to 2010, we collected 10 cases of ovarian cancer patients at Linkou Chang Gung Memorial Hospital, Taiwan. This study was approved by the Institutional Review Board of the Chang Gung Memorial Hospital, Taiwan (100-3902A3 \& 103-4220C), and the informed consent are obtained from all the patients. The characteristics of the 10 patients are listed in Table 1 . All these ovarian cancer patients received surgery. Among the 10 cases, five cases had Seprafilm $(15 \mathrm{~cm}$ $\times 13 \mathrm{~cm}$, genzyme) placed during surgery, and the other five cases don't have Seprafilm placed during surgery.

Considering the natural absorption of Seprafilm (that is, Seprafilm will form a gel-like fluid within $24 \mathrm{~h}$ to $48 \mathrm{~h}$, and slowly resorbed within 7 days), we check immune risk profiles (IRP) before the operation, and immune risk profiles one week later after the operation. The immune risk profiles we checked included CD3 (common T cells), CD19 (B cells), CD4, CD8, CD40, CD28, CD154, NK, and immunoregulatory $\mathrm{CD} 25$ and $\mathrm{CD} 11 \mathrm{~b}$, etc. The statistic difference of selected immune risk profiles $(\mathrm{CD} 4, \mathrm{CD} 8, \mathrm{NK}, \mathrm{CD} 11 \mathrm{~b}, \mathrm{CD} 28)$ between two groups of patients were analyzed by Chi-square.

We also check some suspected markers of other danger signals before operation and one week after operation. Uric acid was chosen as

\begin{tabular}{|l|l|c|c|}
\hline \multirow{3}{*}{ Age } & Range & Seprafilm & Non-Seprafilm \\
\cline { 2 - 4 } & Median & $23-61$ & $50-57$ \\
\hline \multirow{5}{*}{ Pathology } & Mucinous & 49.4 & 53.8 \\
\cline { 2 - 4 } & Serous & 1 & 1 \\
\cline { 2 - 4 } & Papillary serous & 2 & 3 \\
\cline { 2 - 4 } & Endometrioid & 1 & 0 \\
\cline { 2 - 4 } & Mixed pattern & 0 & 0 \\
\hline \multirow{5}{*}{ Stage } & I/II & 1 & 1 \\
\cline { 2 - 4 } & III/IV & 4 & 0 \\
\hline \multirow{5}{*}{ Surgery } & Debulking & 1 & 5 \\
\cline { 2 - 4 } & Fertility preserving & 1 & 0 \\
\cline { 2 - 4 } & Inoperable & 3 & 1 \\
\cline { 2 - 4 } & Second look & 3.8 & 1 \\
\hline Survival & $>5$ years & & 3 \\
\cline { 2 - 4 } & Median survival (year) & 1 & 0 \\
\hline
\end{tabular}

Table 1: Patient characteristics.

classic danger signal. CRP, LDH and d-dimer were chosen as marker to monitor homeostatic danger signal.

\section{Results}

\section{Characteristics of the patients}

The characteristics of the 10 cases of ovarian cancer is listed in Table

*Corresponding author: Cheng-Tao Lin, Department of Obstetrics and Gynecology, Chang Gung Memorial Hospital, Linkou Medical Center, Chang Gung University College of Medicine, 5, Fu-Hsin Street, Kwei Shan Taoyuan, Taiwan, Tel: 886-3-3281200; E-mail: 51424@cgmh.org.tw

Received September 20, 2016; Accepted September 23, 2016; Published September 30, 2016

Citation: Tzu-Hsuan C, Hsiu-Huei P, Cheng-Tao L (2016) Seprafilm Mediated Immune Response Mimic Danger Signal: A Pilot Study in Ovarian Cancer Cases. J Nucl Med Radiat Ther 7: 305. doi: 10.4172/2155-9619.1000305

Copyright: (C) 2016 Tzu-Hsuan C, et al. This is an open-access article distributed under the terms of the Creative Commons Attribution License, which permits unrestricted use, distribution, and reproduction in any medium, provided the original author and source are credited. 
Citation: Tzu-Hsuan C, Hsiu-Huei P, Cheng-Tao L (2016) Seprafilm Mediated Immune Response Mimic Danger Signal: A Pilot Study in Ovarian Cancer Cases. J Nucl Med Radiat Ther 7: 305. doi: 10.4172/2155-9619.1000305

Page 2 of 3

1. In Seprafilm group (SG), the median age was $49.4 \mathrm{y} / \mathrm{o}$ and only one case was stage Ic ovarian cancer, the other 4 cases were all advanced stage. In non-Seprafilm group (NSG), the median age was $53.8 \mathrm{y} / \mathrm{o}$ and all of the 5 cases has advanced stage ovarian cancer. In 5-year-survival, 3 patients in Seprafilm group were over 5 years and none in nonSeprafilm group had survival over 5 years.

\section{Immune risk profiles}

The immune risk profiles (CD4, CD8, NK, CD11b, CD28) before operation and one week after operation in 5 cases of Seprafilm group (SG) and 5 cases of non-Seprafilm group (NSG) are shown in Table 2. Elevated CD4 in $80 \%$ cases $(4 / 5)$ and elevated NK in $60 \%$ cases $(3 / 5)$ were noted in Seprafilm group. In the non-Seprafilm group, elevated CD4 in $60 \%$ cases $(3 / 5)$ and elevated NK in $20 \%$ cases $(1 / 5)$ were noted, respectively. We observed that CD4 had an increasing trend in Seprafilm group compared to non-Seprafilm group, although it doesn't has statistical significance. Moreover, we find out CD4 helper T cells to be elevated $5.1 \%$ (range $2.4 \%$ to $9 \%$ ) in 4 cases of Seprafilm group one week after operation, compared to $4.6 \%$ (range $2 \%$ to $7.2 \%$ ) in three cases of non-Seprafilm group.

CD28 elevation had same cases in both groups, that is, $60 \%(3 / 5)$ in Seprafilm group and 60\% (3/5) in non-Seprafilm group. Also, we find out less CD28 percentage to elevate $5.1 \%$ (range $0.5 \%$ to $10.3 \%$ ) in Seprafilm group than $7.6 \%$ (range $7.4 \%$ to $8.6 \%$ ) in non-Seprafilm group. These findings indicate that operative insertion of Seprafilm may rendered host's immune cells polarized less Th2 status to enhance more Th1 status.

\section{Uric acid, CRP, LDH and d-dimer}

The result of uric acid, CRP, LDH and d-dimer before operation and one week after operation in 5 cases of Seprafilm group (SG) and 5 cases of non-Seprafilm group (NSG) are shown in Table 3.1-3.4. We found elevated uric acid in all patients who survived over 5 years (Table 3.1). While, CRP and LDH couldn't found the relation with prognosis in our limited cases (Table 3.2 and 3.3). D-dimer elevation were noted in all patient in non-Seprafilm group (Table 3.4).

\begin{tabular}{|c|c|c|c|}
\hline $\begin{array}{c}\text { Case number of elevated immune } \\
\text { profile }\end{array}$ & Seprafilm & Non-seprafilm & P value \\
\hline CD4 & 4 & 2 & 0.5186 \\
\hline CD8 & 4 & 4 & 1 \\
\hline CD4/CD8 & 3 & 2 & 0.5271 \\
\hline NK & 3 & 1 & 0.5186 \\
\hline CD11b & 3 & 3 & 1 \\
\hline CD28 & 3 & 3 & 1 \\
\hline
\end{tabular}

Table 2: Compare immune profile between Seprafilm group(SG) and non-Seprafilm group(NSG).

\begin{tabular}{|c|c|c|c|}
\hline & UA before operation & UA after operation & \\
\hline S1 & 5.6 & 8.6 & +3 \\
\hline S2 & 5 & 6.3 & +1.3 \\
\hline S3 & 5 & 4.6 & -0.4 \\
\hline S4 & 3.1 & 6.3 & +3.2 \\
\hline S5 & 3 & 3.6 & +0.6 \\
\hline NS1 & 6.6 & 9.6 & +3 \\
\hline NS2 & 3.5 & 4.7 & +1.2 \\
\hline NS3 & 3.8 & 9.2 & +5.4 \\
\hline NS4 & 5.7 & NA & NA \\
\hline NS5 & 8 & 3.2 & -4.8 \\
\hline
\end{tabular}

Table 3.1: Compare uric acid change between SG and NSG.

\begin{tabular}{|c|c|c|c|}
\hline & CRP before operation & CRP after operation & \\
\hline S1 & 0.58 & 3.89 & +3.31 \\
\hline S2 & 20.9 & 161.26 & +140.36 \\
\hline S3 & NA & 33.52 & NA \\
\hline S4 & 87.95 & 18.36 & -69.59 \\
\hline S5 & 2.01 & 9.04 & +7.03 \\
\hline NS1 & 357.87 & NA & NA \\
\hline NS2 & 114.35 & 37.58 & -76.77 \\
\hline NS3 & 41.03 & 1.85 & -39.18 \\
\hline NS4 & 8.05 & 90.43 & +82.38 \\
\hline NS5 & 28.89 & 38.26 & +9.38 \\
\hline
\end{tabular}

Table 3.2: Compare C-reactive protein(CRP) change between SG and NSG.

\begin{tabular}{|c|c|c|c|}
\hline & LDH before operation & LDH after operation & \\
\hline S1 & NA & 529 & NA \\
\hline S2 & 278 & 301 & +23 \\
\hline S3 & 359 & 335 & -24 \\
\hline S4 & 271 & 389 & +118 \\
\hline S5 & 437 & 251 & -186 \\
\hline NS1 & 1529 & 896 & -633 \\
\hline NS2 & 616 & 332 & -284 \\
\hline NS3 & 265 & 240 & -25 \\
\hline NS4 & 136 & NA & NA \\
\hline NS5 & 450 & 414 & -36 \\
\hline
\end{tabular}

Table 3.3: Compare lactic dehydrogenase(LDH) change between SG and NSG.

\begin{tabular}{|c|c|c|c|}
\hline & D-dimer before operation & D-dimer after operation & \\
\hline S1 & 204 & 1057 & +853 \\
\hline S2 & 3636 & 2130 & -1506 \\
\hline S3 & 2505 & $>10000$ & $>+7495$ \\
\hline S4 & $>10000$ & 4639 & $<-5361$ \\
\hline S5 & 481 & 3302 & +2821 \\
\hline NS1 & 8929 & $>10000$ & $>+1071$ \\
\hline NS2 & 2943 & 3940 & +997 \\
\hline NS3 & 3807 & 4270 & +463 \\
\hline NS4 & 4103 & $>10000$ & $>+5897$ \\
\hline NS5 & 2415 & 6083 & +3668 \\
\hline
\end{tabular}

Table 3.4: Compare D-dimer change between SG and NSG.

\section{Discussion}

Several studies had shown that the immune system plays an important role in controlling and elimination of cancer. T-cell rich ovarian tumors were associated with improved 5 -year overall survival relative to T-cell poor ovarian tumors $(38.0 \%$ vs. $4.5 \%)$ [2]. In general, the immunocompromised patients had more risks of cancer relapse following conventional therapy. The existence of local residual cancer cells resulted from escaping recognition of host immunosurveillance. Therefore, improving existing host immune systemic scenarios through immunomodulatory adjuvants was needed.

Harnessing immune cells switched Th1/Th2 responses in relapse of ovarian cancer that all signals were needed [3]. After conventional optimal debulking operation, we tried to find a way to trigger antigenpresenting cells such as dendtritic cells, macrophages and B cells which would generate and/or amplify host immunosurveillance. The final goal is to improve overall survival, long-term survival rate and prevent relapse of ovarian cancer.

The term "danger signal" was proposed in 1994. It's an alarm signal sent by dead, damaged or invaded cell, which included exogenous and endogenous origin. In response to danger signal, dendritic cells "the 
Citation: Tzu-Hsuan C, Hsiu-Huei P, Cheng-Tao L (2016) Seprafilm Mediated Immune Response Mimic Danger Signal: A Pilot Study in Ovarian Cancer Cases. J Nucl Med Radiat Ther 7: 305. doi: 10.4172/2155-9619.1000305

director of the T-cell immune system" would be matured and further enhance T-cell response. For naïve T-cell activation, the danger signal mimic signal 0 to facilitate dendritic cells. Based on the above theory, we hoped to find a substance we could apply on increasing T-cell response. In the past, hyaluronan had been reported as one of endogenous danger signals $[4,5]$. Seprafilm which is a hyaluronate-based agent was used for preventing postoperative adhesion. The Seprafilm hydrates to form a gel-like fluid within $24 \mathrm{~h}$ to $48 \mathrm{~h}$, and is slowly resorbed within 7 days [6]. Our hypothesis is that additional Seprafilm placed during operation could mimic danger signal and induce intraperitoneal immune response. If further proved, it might be an additional management option for ovarian cancer patients, which had 5-year-survival rate around $50 \%$ and even worse in advanced stage [7].

In our study, we observed that CD4 had an increasing trend in Seprafilm group compared to non-Seprafilm group, although it doesn't has statistical significance. The results indicate that Seprafilm may mimic danger signal to activate more dendritic cells. Later, matured DCs could be initiators of immune responses like stimulation of quiescent, naive and memory, B and T lymphocytes [8-11]. Effector T-cell such as cytotoxic T-cell had role in destroying cancer cell. Memory effector cell could play a part in protecting our body from cancer cell recurrence. Naïve $T$ cells are activated in secondary lymphoid tissue by dendritic cells presenting cognate antigen and providing co-stimulation. In all these effector T cells, approximately $90 \%$ to $95 \%$ will die over 1-2 weeks. Other $5 \%$ to $10 \%$ of the antigen-specific T-cell population survives and maintained indefinitely. That means, few activated $\mathrm{T}$ cells generate fewer effector $\mathrm{T}$ cells, much fewer memory $\mathrm{T}$ cells. Based on this, if we can activate more dendritic cells, we will activate more effector $\mathrm{T}$ cells and memory $\mathrm{T}$ cells (Figure 1).

In the previous study, Ruiz-Perez et al. had reported that Seprafilm as a immunomodulator in sepsis (animal model) [12-14]. It had showed a trend in suppression of T-cell proliferation and reduction in their ability to release IL-2 and TNF-a. Our study shows similar result, that is, signal $2 \mathrm{CD} 28$ had less elevate mean of immune cells index in Seprafilm group than non-Seprafilm group.

As we know, there are three kinds of danger signals. First, classic danger signal, refer to pathogen-associated molecular patterns (PAMPs) or directly derived from tissue injury damage-associated molecular patterns (DAMPs), or secreted by activated immune cells. Second, emerging danger signal, include inorganic materials and man-made technologies. Third, homeostatic danger signal, represent perturbations in tissue steady state resulting inflammation. In our study, Seprafilm could be regarded as our "candidate" of emerging danger signal. We also collected uric acid, CRP, LDH and d-dimer as suspected clinical

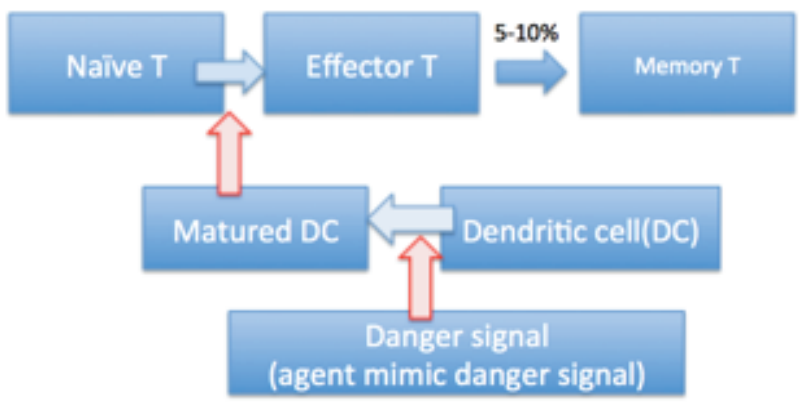

Figure 1: The danger signal can induce immature dendritic cell to mature dendritic cell. danger signal marker. We found elevated uric acid in all patients who survived over 5 years (Table 3.1), and D-dimer elevation in all patient in non-Seprafilm group (Table 3.4). We supposed uric acid could be regarded as clinical monitor of danger signal, and elevated d-dimer may relate to poor prognosis.

In conclusion, we believed that Seprafilm could be an optional agent mimic danger signal. Reliable clinical markers to monitor danger signal can assist in monitoring efficacy of immunotherapy, and uric acid may be a useful marker. Further studies with larger sample size and cytokine profiles were needed, which is important for improving survival rate and prevent cancer relapse.

\section{Acknowledgments}

The study was supported by grants CRRPG3C0911, CRRPG3D0051-3, CMRPG3F0251 (Cheng-Tao Lin), which was provided by Chang Gung Memorial Hospital (Taoyuan, Taiwan).

\section{References}

1. Cannistra SA (2004) Cancer of the ovary. N Engl J Med 351: 2519-2529.

2. Zhang L, Conejo-Garcia JR, Katsaros D, Gimotty PA (2003) Intratumoral T Cells, Recurrence and Survival in Epithelial Ovarian Cancer. N Engl J Med 348: 203-213.

3. Lin CT (2016) Harnessing immune switch responses in relpase of ovarian cancer : all signals needed. J Nucl Med Radiat Ther 7: 286.

4. Matzinger $P$ (1994) Tolerance danger and the extended family. Annu Rev Immunol 12: 991-1045.

5. Scheibner KA, Lutz MA, Boodoo S, Fenton MJ, Powell JD, et al. (2006) Hyaluronan Fragments Act as an Endogenous Danger Signal by Engaging TLR2. J Immunol 177: 1272-1281.

6. Termeer CC, Hennies J, Voith U, Ahrens T, Weiss JM, et al. (2000) Oligosaccharides of Hyaluronan Are Potent Activators of Dendritic Cells. Immunol. 165: 1863-1870.

7. Reijnen MM, Bleichrodt RP, van Goor H (2003) Pathophysiology of intraabdominal adhesion and abscess formation, and the effect of hyaluronan. $\mathrm{Br}$ J Surg 90: 533-541.

8. Ojalvo LS, Niclos PE, Jelovac D, Emens LA (2015) Emerging Immunotherapies in Ovarian Cancer. Discov Med 20: 97-109.

9. Banchereau J, Steinman RM (1998) Dendritic cells and the control of immunity. Nature 392: 245-252.

10. Masopust D, Kaech SM, Wherry EJ, Ahmed R (2004) The role of programming in memory T-cell development. Curr Opin Immunol 16: 217-225.

11. Gallucci S, Matzinger P (2001) Danger signals: SOS to the immue system. Curr Opin Immunol 13: 114-119.

12. Borrello I, Pardoll D (2002) GM-CSF-based cellular vaccines: a review of the clinical experience. Cytokine Growth Factor Rev 13: 185-193.

13. Ruiz-Perez B, Cisneros RL, Matsumoto T, Miller RJ, Vasios G, et al. (2003) Protection against Lethal Intra-abdominal Sepsis by 1-(3-dimethylaminopropyl)3-ethylurea. J Infect Dis 188: 378-387

14. Reijnen MMPJ, Meis JFGM, Postma VA, van Goor H (1999) Prevention of intraabdominal abscesses and adhesions using a hyaluronic acid solution in a rat peritonitis model. Arch Surg 134: 997-1001. 